\title{
Routine left atrial appendage ligation during cardiac surgery may prevent postoperative atrial fibrillation-related cerebrovascular accident
}

\author{
Ryan Kim, MD, ${ }^{\mathrm{a}}$ Norbert Baumgartner, MD, FACS, ${ }^{\mathrm{b}}$ and John Clements, $\mathrm{MPA}^{\mathrm{c}}$
}

Objective: The aim of the study was to determine whether routine left atrial appendage ligation in cardiac surgery would reduce the risk of postoperative atrial fibrillation-related cerebrovascular accident.

\begin{abstract}
Methods: We performed an institutional review board-approved, case-control, retrospective chart review of adult patients who underwent cardiac surgery by a single surgeon between January 1, 2001, and December 31, 2010. Preoperative $\mathrm{CHADS}_{2}$ score criteria were collected, and outcomes through postoperative day 30 were analyzed.

Results: A total of 2067 patients were reviewed. Propensity score matching was used to create matched groups based on left atrial appendage ligation, and 631 patients were matched in each group. Subjects with postoperative atrial fibrillation were 2.4 times (95\% confidence interval, 1.51-2.82) more likely to undergo valve surgery, 2.11 times ( $95 \%$ confidence interval, 1.56-2.86) more likely to be aged more than 75 years, and 1.36 times (95\% confidence interval, 1.03-1.80) more likely to have undergone left atrial appendage ligation. In the left atrial appendage ligation group with postoperative atrial fibrillation $(n=145)$, there were zero subjects $(0 \%)$ with a postoperative cardiovascular accident. In the non-left atrial appendage ligation group with postoperative atrial fibrillation $(\mathrm{n}=115)$, there were 7 subjects $(6.1 \%)$ with a postoperative cardiovascular accident $(0.0 \%$ vs $6.1 \%, P=.003)$.
\end{abstract}

Conclusions: Although postoperative atrial fibrillation remains a common complication of cardiac surgery, there was a significant decrease in the incidence of postoperative cerebrovascular accidents since routine ligation of the left atrial appendage was undertaken. This has been found to be a safe adjunct to a wide variety of cardiac procedures and therefore deserves further study by a larger randomized controlled trial. (J Thorac Cardiovasc Surg 2013;145:582-9)

Postoperative atrial fibrillation (AF) is a common complication that occurs in up to $50 \%$ of patients who undergo cardiac surgery, including coronary artery bypass grafting $(\mathrm{CABG})$ and valve procedures. ${ }^{1-6}$ Postoperative AF may present as a benign, transient, and self-limiting arrhythmia with no significant consequences after the surgery; however, it may also result in adverse effects such as tachycardia, hypotension, heart failure, and cerebrovascular accidents (CVAs). ${ }^{1,6,7}$ Several studies have observed embolic episodes and postoperative CVA occurring in patients with postoperative $\mathrm{AF}$, and the emboli are

From the Department of Surgery, ${ }^{\text {a }}$ Central Michigan University Healthcare, Central Michigan University College of Medicine, Saginaw, Mich; Sparrow Healthcare \& Michigan State University College of Human Medicine, ${ }^{\mathrm{b}}$ Lansing, Mich; and Department of Research, ${ }^{\mathrm{c}}$ Central Michigan University Healthcare, Central Michigan University College of Medicine, Saginaw, Mich.

Disclosures: Authors have nothing to disclose with regard to commercial support.

Read at the 38th Annual Meeting of The Western Thoracic Surgical Association, Maui, Hawaii, June 27-30, 2012.

Received for publication May 25, 2012; revisions received Sept 20, 2012; accepted for publication Oct 2, 2012; available ahead of print Nov 7, 2012.

Address for reprints: Ryan Kim, MD, Department of Surgery, Central Michigan University Healthcare, 1000 Houghton Ave, Saginaw, MI 48602 (E-mail: ryan.kim@ cmich.edu)

0022-5223/\$36.00

Copyright (c) 2013 by The American Association for Thoracic Surgery

http://dx.doi.org/10.1016/j.jtcvs.2012.10.016 thought to arise from the thrombus formed primarily in the left atrial appendage (LAA). ${ }^{4,8-10}$

Theoretically, the complete obliteration of the LAA should decrease the incidence of embolic events in patients with AF. Maze and corridor procedures that include LAA exclusion are often used in patients with preoperative $\mathrm{AF}$ in an effort to reduce the risk of embolic stroke ${ }^{8,11,12}$; however, LAA ligation is only routinely performed by most surgeons during mitral valve procedures. ${ }^{4,8}$ There are no studies to date documenting routine LAA ligation in all types of adult cardiac surgery. If LAA exclusion can be shown to reduce the risk of embolic stroke in patients with postoperative $\mathrm{AF}$, it may be an important adjunct to all types of cardiac procedures to reduce the risk of postoperative AF-related CVA.

A small number of patients every year have a postoperative CVA after developing postoperative AF, even when they initially wake up from anesthesia neurologically intact. Since routine LAA exclusion has been implemented in one of the author's practice (N.B.), the incidence of postoperative AF-related CVA seems to have decreased. This study was undertaken to determine whether routine LAA ligation in cardiac surgery reduces the risk of postoperative AF-related postoperative CVA. 


\section{Abbreviations and Acronyms \\ $\mathrm{AF}=$ atrial fibrillation \\ CABG = coronary artery bypass grafting \\ $\mathrm{CHADS}_{2}=\mathrm{C}$ : congestive heart failure, $\mathrm{H}$ : \\ hypertension, A: age $>75$ years, D: \\ diabetes, S: stroke [stroke assessment risk] \\ CHF = congestive heart failure \\ $\mathrm{CI}=$ confidence interval \\ CVA = cerebrovascular accident \\ LAA $=$ left atrial appendage \\ PSM = propensity score matching}

\section{MATERIALS AND METHODS}

\section{Study Design}

This was approved by the hospitals' institutional review boards. Charts of all patients who underwent cardiac surgery with a single cardiothoracic surgeon from January 1, 2001, to December 31, 2010, were reviewed. In mid-2003, after the observation of a significant number of postoperative AF-related CVAs, there was a change in this surgeon's (N.B.) standard practice to include exclusion, and later excision, of the LAA. As such, 2 groups based on those who did and did not receive LAA ligation were formed. Patients with incomplete medical records and patients who died during the surgery were excluded. Patients' medical records were reviewed up to 30 days postoperatively.

\section{Surgical Management}

A wide variety of adult cardiac procedures were performed during this period, both on-pump and off-pump, most of them via median sternotomies. In cases in which the aorta was crossclamped, the LAA was ligated at the base, flush with the left atrial wall, with 2 layers of running nonabsorbable monofilament suture. Later in the series, the LAA was excised and the stump was oversewn in 2 layers as described. In cases in which the aorta was not clamped, the LAA was closed at its base with a linear dividing surgical stapler with bioabsorbable reinforcing strips. After reversal of anticoagulation, the LAA site was inspected, and if necessary additional pledgeted sutures were placed to achieve hemostasis. In cases that required opening the left atrium, the LAA ligation was performed first, and the adequacy of the closure was inspected from within the left atrium.

\section{Patients and Outcomes}

Patients' age at the time of surgery, gender, preoperative history of congestive heart failure (CHF), hypertension, age risk, diabetes, and history of stroke (hemorrhagic or thromboembolic) were recorded, and a $\mathrm{CHADS}_{2}$ score (stroke risk assessment in atrial fibrillation: $\mathrm{C}$, congestive heart failure; $\mathrm{H}$, hypertension; $\mathrm{A}$, age $>75$ years; $\mathrm{D}$, diabetes; $\mathrm{S}$, stroke) was calculated for each patient. For history of CHF, patients with New York Heart Association score greater than or equal to 2 and any documented incidence of CHF 1 month before the surgery were classified as having a positive history. The type of surgery performed, occurrence of postoperative AF, and occurrence of postoperative CVA were recorded postoperatively. Patients' medical records were examined through postoperative day 30 for the incidence of postoperative $\mathrm{AF}$ and CVA. All data were obtained from the patients' hospital and office medical records, and the hospital's Society of Thoracic Surgeons cardiac database.

\section{Statistical Analysis}

The Statistical Package for the Social Sciences version 20.0 (SPSS Inc, Chicago, Ill) was used for data analysis. The Pearson chi-square or Fisher exact test was used to assess differences in categoric variables, and the $t$ test was used to determine differences in continuous variables between treatment groups.

Because of the observational nature of this study, treatment groups were not randomly assigned. Propensity score matching (PSM) was therefore used to create matched groups based on treatment. The propensity score was calculated using binary logistic regression models in which LAA ligation therapy was the binary dependent variable. The predicted probability of receiving the treatment group was used as the propensity score. On the basis of this score, each patient who received LAA ligation was matched to a control patient who did not receive LAA ligation. When there were more control subjects who matched a treatment subject on the basis of the propensity score, a random assignment of controls to treatment subjects was made using a random number generator. After matching, the chi-square or Fisher exact test was used to determine the differences in categoric

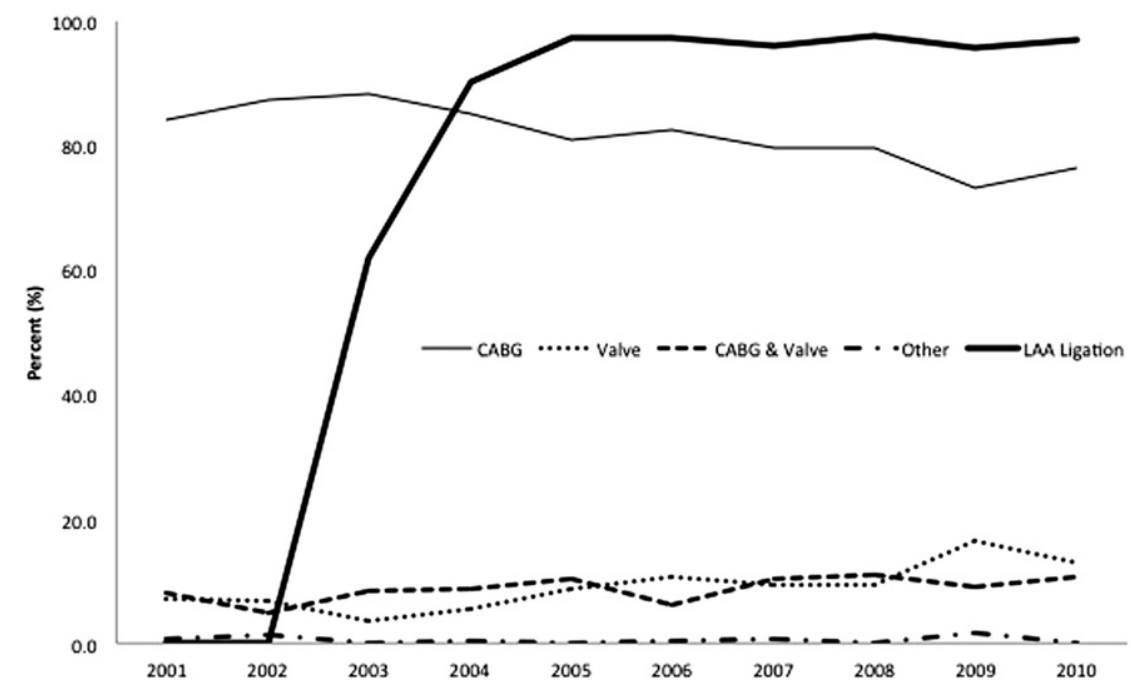

FIGURE 1. Cardiac procedures (2001-2010). $C A B G$, Coronary artery bypass grafting; $L A A$, left atrial appendage. 


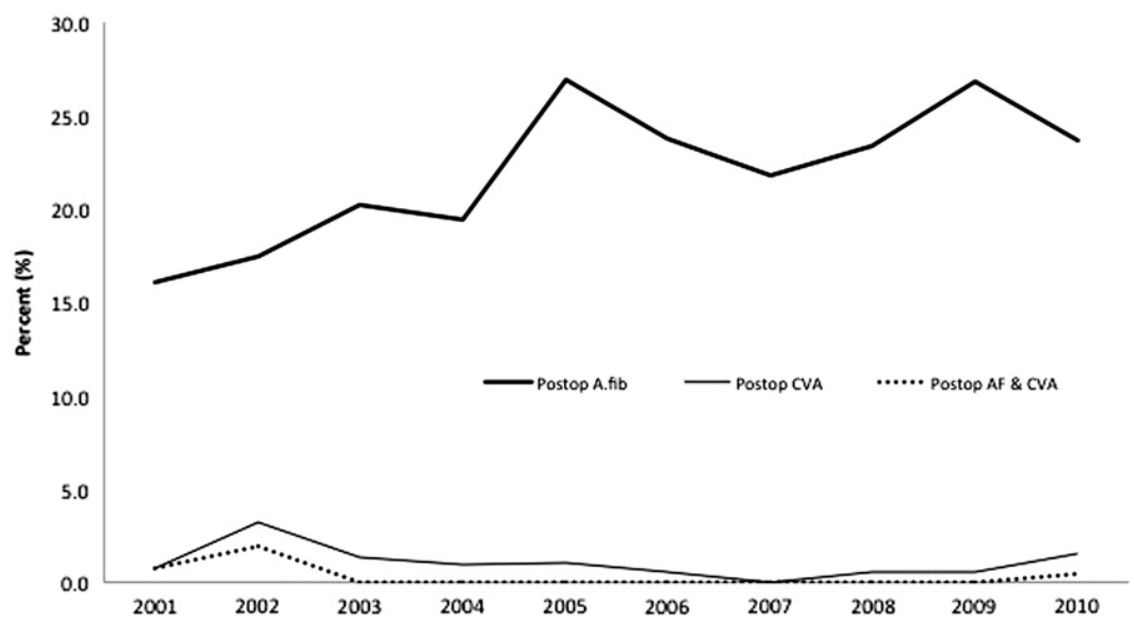

FIGURE 2. Postoperative AF and CVA (2001-2010). AF, Atrial fibrillation; A.fib, atrial fibrillation; CVA, cardiovascular accident.

variables, and a $t$ test was used to measure differences in continuous variables between the matched groups. Finally, binary logistic regression techniques were used to determine which variables were predictive of postoperative $\mathrm{AF}$ and CVA.

\section{RESULTS}

A total of 2078 patients underwent cardiac surgery during the 10-year study time period. Eleven patients were excluded from the study (10 patients died and 1 patient had an incomplete medical record because of transfer to another facility on postoperative day 1), leaving a sample size of 2067. Surgeries included CABG alone in 1696 patients $(82.1 \%)$, valve surgery alone in 182 patients $(8.8 \%)$, combined CABG and valve surgery in 178 patients $(8.6 \%)$, and other cardiac procedures in 11 patients $(0.5 \%)$, such as atrial septal defect repair, ventricular septal defect repair, and atrial myxoma excision.

A few trends were noted over the 10-year period: The total yearly number of cardiac surgery cases decreased, the number of $\mathrm{CABG}$ procedures decreased and the number of valve procedures increased, and the number of cases in which routine LAA ligation was performed increased (Figure 1). Furthermore, the overall yearly incidence of postoperative AF increased and the overall incidence of postoperative CVA decreased, and the incidence of postoperative $\mathrm{AF}$ with postoperative CVA decreased (Figure 2).

Patient ages ranged from 21 to 94 years, with a mean age of $66.4 \pm 11.5$ years. A total of 665 patients $(32.3 \%)$ were female, 1465 patients $(70.9 \%)$ had a history of CHF, 1620 patients $(78.4 \%)$ had a history of hypertension, 573 patients $(27.7 \%)$ were aged 75 years or more, 713 patients $(34.5 \%)$ had a history of diabetes, and 158 patients $(7.6 \%)$ had a history of CVA. $\mathrm{CHADS}_{2}$ score ranged from zero to 6 , with a mean of $2.27 \pm 1.1$ and mode of 2 (748 [36.2\%]). During the 10-year period, there were 445 patients $(21.5 \%)$ with postoperative $\mathrm{AF}$ and 23 patients $(1.1 \%)$ with postoperative CVA. All postoperative CVAs were confirmed with computed tomography of the brain, magnetic resonance imaging of the brain, or cerebral angiogram. Transient ischemic attacks were not included as positive postoperative CVAs. The causes of the postoperative CVA were extracranial carotid disease (5 patients), paradoxical embolism from a patent foramen ovale (1 patient), surgical manipulation of the aorta (1 patient), septic emboli (1 patient), and thromboembolic event of unknown origin (16 patients). Only 1 patient had a postoperative CVA immediately after waking up from the surgery, which was believed to be secondary to embolism of aortic atheroma. Eight subjects $(0.4 \%)$ had both postoperative AF and postoperative CVA (Table 1). Two subjects underwent CABG surgery alone, 3 subjects underwent valve surgery alone, and 3 subjects

TABLE 1. Baseline characteristics of study patients

\begin{tabular}{lccc}
\hline & Entire group & Ligation & Nonligation \\
\hline Total no. & 2067 & 1405 & 662 \\
Age (y) & $66.4 \pm 11.5$ & $66.6 \pm 11.4$ & $65.8 \pm 11.6$ \\
Gender (male/female) & $1402 / 665$ & $954 / 451$ & $447 / 214$ \\
CABG only n (\%) & $1696(82.1)$ & $1143(81.4)$ & $553(83.5)$ \\
Valve surgery only n (\%) & $182(8.8)$ & $130(9.2)$ & $52(7.9)$ \\
CABG and valve surgery n (\%) & $178(8.6)$ & $126(9.0)$ & $52(7.9)$ \\
Other open surgery n (\%) & $11(0.5)$ & $6(0.4)$ & $5(0.7)$ \\
History of CHF n (\%) & $1465(70.9)$ & $933(66.4)$ & $532(80.4)$ \\
History of HTN n (\%) & $1620(78.4)$ & $1136(80.9)$ & $484(73.1)$ \\
Age $>75$ y n (\%) & $573(27.7)$ & $392(27.9)$ & $181(27.3)$ \\
History of diabetes n (\%) & $713(34.5)$ & $479(34.1)$ & $234(35.3)$ \\
History of stroke n (\%) & $158(7.6)$ & $114(8.1)$ & $44(6.6)$ \\
CHADS 2 mean score & $2.27 \pm 1.12$ & $2.25 \pm 1.14$ & $2.29 \pm 1.07$ \\
Postoperative AF n (\%) & $445(21.5)$ & $323(23.0)$ & $122(18.4)$ \\
Postoperative CVA n (\%) & $23(1.1)$ & $13(0.9)$ & $10(1.5)$ \\
Postoperative AF and & $8(0.4)$ & $1(0.1)$ & $7(1.1)$ \\
postoperative CVA n (\%) & & & \\
\hline
\end{tabular}

$C A B G$, Coronary artery bypass grafting; $C H F$, congestive heart failure; $H T N$, hypertension; $\mathrm{CHADS}_{2}$, C: congestive heart failure, $\mathrm{H}$ : hypertension, A: age $>75$ years, D: diabetes, $\mathrm{S}$ : stroke; $A F$, atrial fibrillation; $C V A$, cerebrovascular accident. 
TABLE 2. Comparison of left atrial appendage groups with and without propensity score matching*

\begin{tabular}{|c|c|c|c|c|c|c|}
\hline & \multicolumn{3}{|c|}{ LAA groups without PSM } & \multicolumn{3}{|c|}{ LAA groups with PSM } \\
\hline & LAA & Non-LAA & $P$ value & LAA & Non-LAA & $P$ value \\
\hline \multicolumn{7}{|l|}{ Variables } \\
\hline \multicolumn{7}{|l|}{ Independent variables } \\
\hline CABG surgery & $1269(0.90)$ & $605(0.91)$ & .44 & $583(0.92)$ & $583(0.92)$ & 1.00 \\
\hline Valve surgery & $256(0.18)$ & $104(0.16)$ & .16 & $96(0.15)$ & $96(0.15)$ & 1.00 \\
\hline $\mathrm{CABG}$ and valve surgery & $126(0.09)$ & $52(0.072)$ & .40 & $48(0.08)$ & $48(0.08)$ & 1.00 \\
\hline Gender (male) & $954(0.68)$ & $447(0.68)$ & .90 & $430(0.68)$ & $430(0.68)$ & 1.00 \\
\hline Age risk & $392(0.28)$ & $181(0.27)$ & .79 & $164(0.26)$ & $164(0.26)$ & 1.00 \\
\hline History of CHF & $933(0.66)$ & $532(0.80)$ & $<.001$ & $508(0.81)$ & $508(0.81)$ & 1.00 \\
\hline History of HTN & $1136(0.81)$ & $484(0.73)$ & $<.001$ & $470(0.75)$ & $470(0.75)$ & 1.00 \\
\hline History of diabetes & $479(0.34)$ & $234(0.35)$ & .57 & $216(0.34)$ & $216(0.34)$ & 1.00 \\
\hline History of stroke & $114(0.08)$ & $55(0.07)$ & .24 & $31(0.05)$ & $31(0.05)$ & 1.00 \\
\hline Age & $66.6 \pm 11.4$ & $65.8 \pm 11.6$ & .16 & $66.2 \pm 11.1$ & $65.7 \pm 11.6$ & .36 \\
\hline Propensity score & $0.69 \pm 0.08$ & $0.66 \pm 0.08$ & $<.001$ & $0.66 \pm 0.08$ & $0.66 \pm 0.08$ & 1.00 \\
\hline \multicolumn{7}{|l|}{ Dependent variables } \\
\hline Postoperative AF & $323(0.23)$ & $122(0.18)$ & .019 & $145(0.23)$ & $115(0.18)$ & .037 \\
\hline Postoperative CVA & $13(0.009)$ & $10(0.015)$ & .24 & $6(0.01)$ & $9(0.01)$ & .44 \\
\hline Postoperative AF with CVA & $1(0.003)$ & $7(0.057)$ & $<.001$ & $0(0.00)$ & $7(0.06)$ & .003 \\
\hline
\end{tabular}

$L A A$, Left atrial appendage; $P S M$, propensity score matching; $C A B G$, coronary artery bypass grafting; $C H F$, congestive heart failure; $H T N$, hypertension; $A F$, atrial fibrillation; $C V A$, cerebrovascular accident. *Mean \pm standard deviation or number (proportion).

underwent combined $\mathrm{CABG}$ and valve surgery. Postoperative AF developed in 5 subjects before postoperative day 5 (postoperative days 2-5), and these subjects experienced mental status changes within 24 to 72 hours after the initial postoperative AF. One subject had acute endocarditis preoperatively, and septic emboli developed before the valve replacement. Postoperative AF also developed in this subject on postoperative day 6 , with worsening mental status on the same day.

The PSM model included 8 variables: CABG procedure, valve replacement, gender, age risk, history of CHF, history of hypertension, history of diabetes, and history of CVA. The propensity score model was found to be reliable
(Hosmer-Lemeshow test $P=.4$ ) and discriminant (c-index, $0.61 ; 95 \%$ confidence interval $[\mathrm{CI}], 0.58-0.63 ; P<.001)$. By using the results of this model, 631 of 1405 subjects undergoing LAA ligation were matched to 631 of 662 subjects not undergoing LAA ligation. Subjects were well matched on the basis of this procedure (Table 2).

Before PSM, LAA groups were dissimilar with regard to history of CHF and hypertension. These differences were nullified after PSM techniques were applied to the data. However, with regard to 2 dependent variables, postoperative $\mathrm{AF}$ and postoperative $\mathrm{AF}$ with $\mathrm{CVA}$, the differences observed before PSM techniques were still evident after PSM techniques were applied to the data (Table 2).

TABLE 3. Logistic regression analyses

\begin{tabular}{|c|c|c|c|c|}
\hline \multirow{3}{*}{$\begin{array}{c}\text { Variable } \\
\text { Model } \mathbf{R}^{2}\end{array}$} & \multicolumn{2}{|c|}{ Model 1: postoperative AF } & \multicolumn{2}{|c|}{ Model 2: postoperative CVA } \\
\hline & \multicolumn{2}{|c|}{0.067} & \multicolumn{2}{|c|}{0.092} \\
\hline & Regression coefficient & Odds ratio $(95 \% \mathrm{CI})$ & Regression coefficient & Odds ratio $(95 \% \mathrm{CI})$ \\
\hline Constant & $-2.11^{*}$ & & $-6.84 *$ & \\
\hline \multicolumn{5}{|l|}{ Variables } \\
\hline CABG surgery & 0.4 & $1.49(0.78-2.82)$ & 0.13 & $1.14(0.19-6.90)$ \\
\hline Valve surgery & $0.87 *$ & $2.38(1.51-3.77)$ & 1.03 & $2.01(0.78-10.1)$ \\
\hline Male gender & -0.02 & $0.98(0.72-1.33)$ & -0.07 & $0.93(0.32-2.72)$ \\
\hline Age $>75 y$ & $0.75^{*}$ & $2.11(1.63-2.86)$ & 0.79 & $2.22(0.74-6.70)$ \\
\hline History of CHF & -0.16 & $0.85(0.60-1.22)$ & 1.08 & $2.93(0.37-23.1)$ \\
\hline History of HTN & 0.02 & $1.02(0.73-1.42)$ & 0.88 & $2.40(0.052-11.0)$ \\
\hline History of diabetes & -0.17 & $0.84(0.62-1.15)$ & 0.21 & $1.23(0.39-3.85)$ \\
\hline History of stroke & 0.49 & $1.63(0.90-2.96)$ & -16.7 & $0(0-0)$ \\
\hline Postoperative AF & & & 1.03 & $2.79(0.95-8.19)$ \\
\hline LAA ligation & $0.31 *$ & $1.36(1.03-1.78)$ & -0.51 & $0.60(0.21-1.73)$ \\
\hline
\end{tabular}


TABLE 4. Subgroup logistic regression analyses

\begin{tabular}{|c|c|c|c|c|}
\hline \multirow{4}{*}{$\frac{\text { Variable }}{\text { Model } \mathbf{R}^{2}}$} & \multicolumn{4}{|c|}{ Subgroup: LAA ligation subjects } \\
\hline & \multicolumn{2}{|c|}{ Model 4: postoperative AF } & \multicolumn{2}{|c|}{ Model 5: postoperative CVA } \\
\hline & \multicolumn{2}{|c|}{0.066} & \multicolumn{2}{|c|}{0.300} \\
\hline & Regression coefficient & Odds ratio $(95 \% \mathrm{CI})$ & Regression coefficient & Odds ratio $(95 \% \mathrm{CI})$ \\
\hline Constant & $-1.98 *$ & & -38.3 & \\
\hline \multicolumn{5}{|l|}{ Variables } \\
\hline CABG & 0.48 & $1.62(0.66-4.02)$ & 16.4 & Not calculated \\
\hline Valve surgery & $0.74^{*}$ & $2.10(1.11-3.97)$ & 0.58 & $1.78(0.16-20.5)$ \\
\hline Male gender & 0.030 & $1.04(0.68-1.57)$ & -0.38 & $0.68(0.12-3.81)$ \\
\hline Age $>75 y$ & $0.83^{*}$ & $2.30(1.52-3.47)$ & $2.04^{*}$ & $7.68(1.25-47.2)$ \\
\hline History of CHF & -0.22 & $0.80(0.50-1.29)$ & 16.1 & Not calculated \\
\hline History of HTN & 0.19 & $1.22(0.77-1.92)$ & -0.85 & $0.43(0.07-2.71)$ \\
\hline History of diabetes & -0.22 & $0.80(0.53-1.23)$ & $2.46^{*}$ & $11.7(1.24-111.2)$ \\
\hline History of stroke & 0.36 & $1.44(0.63-3.28)$ & -16.3 & Not calculated \\
\hline Postoperative AF & & & -16.84 & Not calculated \\
\hline
\end{tabular}

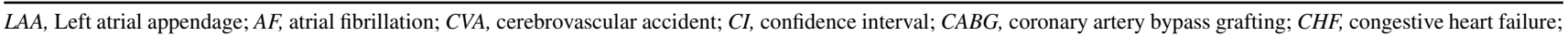
$H T N$, hypertension; $A F$, atrial fibrillation. *Significant at $P<.05$.

After PSM techniques and case-control matching, various regression models were constructed (Table 3) to determine the effects of LAA ligation, as well as the effects of other control variables on the dependent variables. Two models used the 631 matched subject pairs to regress the dependent variables postoperative $\mathrm{AF}$ (model 1 ) and postoperative CVA (model 2). The postoperative AF with CVA case could not be regressed because the number of cases was too small. Regression results for model 1 indicate that subjects with postoperative $\mathrm{AF}$ were 2.4 times $(95 \% \mathrm{CI}, 1.51-2.82$ ) more likely to have valve surgery, 2.11 times $(95 \% \mathrm{CI}$, $1.56-2.86)$ more likely to be aged more than 75 years, and 1.36 times (95\% CI, 1.03-1.80) more likely to have had LAA ligation. Regression results for model 2 (postoperative CVA) indicate there are no significant predictors for postoperative CVA in the matched subject groups.

Subgroup regression analyses also were performed on 3 separate groups (Table 4): the LAA ligation group, nonLAA ligation group, and postoperative AF group. Postoperative $\mathrm{AF}$ and postoperative CVA were regressed in the LAA ligation group (models 4 and 5, respectively) and the nonLAA ligation groups (models 6 and 7, respectively). In addition, postoperative CVA was regressed in the postoperative AF group (model 8).

For the subgroup analyses, logistic regression indicates that in the LAA ligation group, subjects with postoperative $\mathrm{AF}$ were 2.1 times $(95 \% \mathrm{CI}, 1.11-3.97)$ more likely to have had valve surgery and 2.3 times (95\% CI, 1.52-3.47) more likely to be at an increased age, whereas subjects with postoperative CVA were 7.7 times (95\% CI, 1.25-47.2) more likely to be at increased age and 11.7 times $(95 \% \mathrm{CI}$, 1.24-111.2) more likely to have had diabetes. Regression results indicated that in the non-LAA ligation group, subjects with postoperative AF were 2.7 times $(95 \% \mathrm{CI}$,
1.44-5.33) more likely to have had valve surgery and 1.91 times (95\% CI, 1.22-3.01) to have age as a risk factor. In the non-LAA ligation group, subjects with postoperative CVA were 9.1 times (95\% CI, 1.30-64.2) more likely to have had valve surgery and 23.9 times (95\% CI, 3.9$144.78, P<.001)$ more likely to have postoperative AF compared with subjects without postoperative CVA.

Finally, a subsample of 260 subjects with postoperative $\mathrm{AF}$ was analyzed. In the LAA ligation group with postoperative $\mathrm{AF}(\mathrm{n}=145), 0$ subjects $(0 \%)$ had postoperative CVA, whereas in the non-LAA ligation group with postoperative $\mathrm{AF}(\mathrm{n}=115), 7$ subjects $(6.1 \%)$ had postoperative CVA $(0.0 \%$ vs $6.1 \%, P=.003$, using the Fisher exact test).

Logistic regression of the postoperative CVA variable in the subset of all subjects from the PSM-derived groups who had postoperative AF indicated that subjects who had postoperative CVA were approximately 65 times more likely (95\% CI, 2.3-1845; $P=.015)$ to have had valve surgery compared with subjects without postoperative CVA. It is worth noting that logistic regression analyses of the postoperative CVA outcome variable on the postoperative AF group, not subjected to PSM techniques, revealed that those subjects with postoperative $\mathrm{AF}$ who also had postoperative CVA were approximately $95 \%$ less likely $(95 \%$ CI, $0.006-$ $0.409 ; P=.005$ ) to have had LAA ligation during open surgery compared with subjects without postoperative CVA.

\section{DISCUSSION}

Postoperative AF is a common complication of cardiac surgery that occurs in up to $50 \%$ of patients. ${ }^{1-6}$ The occurrence of postoperative AF peaks on postoperative day 2 and usually occurs within 3 to 4 days, with $95 \%$ of cases occurring before day 5., ${ }^{2,3}$ Some researchers regard postoperative AF as a benign, transient, and self-limiting 
TABLE 4. Continued

\begin{tabular}{|c|c|c|c|c|c|}
\hline \multicolumn{4}{|c|}{ Subgroup: non-LAA ligation subjects } & \multirow{2}{*}{\multicolumn{2}{|c|}{$\begin{array}{l}\text { Subgroup: postoperative AF subjects } \\
\text { Model 8: postoperative CVA }\end{array}$}} \\
\hline \multicolumn{2}{|c|}{ Model 6: postoperative AF } & \multicolumn{2}{|c|}{ Model 7: postoperative CVA } & & \\
\hline \multicolumn{2}{|c|}{0.065} & \multicolumn{2}{|c|}{0.420} & \multicolumn{2}{|c|}{0.550} \\
\hline Regression coefficient & Odds ratio $(95 \% \mathrm{CI})$ & Regression coefficient & Odds ratio $(95 \% \mathrm{CI})$ & Regression coefficient & Odds ratio $(95 \% \mathrm{CI})$ \\
\hline$-1.93 *$ & & -26.3 & & -27.3 & \\
\hline 0.32 & $1.37(0.55-3.38)$ & 0.89 & $2.42(0.29-20.5)$ & 1.52 & $4.58(0.40-52.7)$ \\
\hline $1.02 *$ & $2.77(1.44-5.33)$ & $2.21 *$ & $9.13(1.30-64.2)$ & $4.17 *$ & $64.6(2.26-1845)$ \\
\hline-0.09 & $0.92(0.59-1.44)$ & 1.12 & $3.07(0.51-18.5)$ & 1.89 & $6.65(0.42-104.2)$ \\
\hline $0.65^{*}$ & $1.91(1.22-3.01)$ & -0.12 & $0.89(0.17-4.48)$ & -0.80 & $0.45(0.05-4.29)$ \\
\hline 0.08 & $0.92(0.54-1.57)$ & 1.25 & $3.47(0.29-41.8)$ & 2.09 & $8.13(0.22-295.1)$ \\
\hline-0.17 & $0.84(0.52-1.36)$ & 18.1 & Not calculated & 19.7 & Not calculated \\
\hline-0.12 & $0.89(0.56-1.41)$ & -17.5 & $0(0-0)$ & -19.3 & $0(0-0)$ \\
\hline \multirow[t]{3}{*}{0.64} & $1.89(0.80-4.45)$ & -16.8 & $0(0-0)$ & -16.8 & $0(0-0)$ \\
\hline & & $3.17 *$ & $23.9(3.93-144.7)$ & & \\
\hline & & & & -19.6 & $0(0-0)$ \\
\hline
\end{tabular}

arrhythmia with no significant consequences. ${ }^{1,6,7}$ However, it is possible that postoperative AF may result in serious adverse events, including tachycardia, hypotension, heart failure, and CVAs. ${ }^{1,6}$

The cause and pathophysiology of postoperative AF are not fully understood. ${ }^{2,3,5,13}$ Some suspect that the preexisting structural changes in the atria and electrophysiologic abnormalities may play a role in the pathogenesis. ${ }^{2,3,13}$ Furthermore, some speculate that the postoperative state itself and its associated inflammatory response would create a thrombogenic environment. 5,14

If and when postoperative AF occurs, these patients are at risk for an embolic episode with subsequent morbidity or mortality. ${ }^{8,9}$ Many would agree that these embolic episodes and postoperative CVA arise from thrombus formed in the LAA. ${ }^{4,9,10}$ Studies have shown that $90 \%$ of atrial clots are formed in the LAA, and the emboli cause approximately $25 \%$ of all strokes. ${ }^{4,9,10}$

Because it is well known that embolic stroke is a complication of $\mathrm{AF}^{6,15}$ the obliteration or occlusion of the LAA should be an effective way to decrease the incidence of embolic stroke in patients with AF. There have been many studies of LAA occlusion in efforts to reduce the risk of strokes in patients with chronic $\mathrm{AF}^{8,11,12}$; however, routine LAA ligation is currently not performed by most cardiac surgeons. ${ }^{4,8}$ The American College of Cardiology/American Hospital Association 2006 guidelines for valvular heart disease recommend amputation of the LAA at the time of mitral valve surgery to reduce the risk of postoperative CVA, ${ }^{10,16}$ but it is not a standard procedure for other cardiac procedures, such as CABG. $^{4,8}$ This study may be the first to document the advantages of routine LAA ligation in all types of cardiac surgery.
Some studies have found that the function of LAA is limited, and its removal would be of benefit in higherrisk patients. ${ }^{4,9}$ Although there are studies indicating the risk of incomplete surgical LAA ligation, ${ }^{17}$ LAA ligation is a safe and feasible procedure., ${ }^{4,11}$ If complete occlusion is achieved, no significant adverse effects from the removal of LAA have been documented in the literature. $^{4,9,11}$

On the basis of these observations, LAA ligation was implemented as a routine part of each cardiac surgical procedure by one of the authors (N.B.) without changing other standards of care. It was hoped that by doing so, the few cases of postoperative CVA in patients who were initially neurologically intact and then developed a stroke after the onset of postoperative AF could be avoided.

Postoperative AF remains a common complication after cardiac surgery. In this study, the overall incidence of postoperative AF was $21.5 \%$, which is similar to rates reported in the literature and the Society of Thoracic Surgeons database. $^{1-6}$ The incidence of postoperative AF ranged from $16.0 \%$ to $26.9 \%$ annually; however, as the statistical analysis indicates, the incidence of postoperative AF increased during the period of routine LAA ligation. As expected, however, the incidence of postoperative CVA decreased as routine LAA ligation was implemented, despite the increased incidence of postoperative AF.

The cause of postoperative AF is still unknown. The current study found that age is a significant risk factor for postoperative AF, as reported in other studies. ${ }^{2,3,13}$ The increased rate of AF in patients who underwent LAA exclusion or excision, which was observed in the current study, may be secondary to local structural changes in the region of the LAA or irritable foci caused by the ligation or exclusion procedure. Another possibility for the increased rate of postoperative 
AF observed in the group that underwent LAA ligation is simply that there was an increased number of valve procedures, which is another factor known to be associated with postoperative AF and confirmed in this analysis.

There were no serious complications associated with the exclusion or excision of the LAA in this series, and no reoperations were required for bleeding from the LAA. In 1 case, an elderly female patient with a friable LAA was undergoing $\mathrm{CABG}$ and an avulsion at the base of the atrial appendage necessitated a left atriotomy and pericardial patch repair of the defect. She recovered uneventfully without sequelae. In all cases in which the atrial appendage was excluded and then inspected from within the left atrium, there was a complete closure of the anatomic orifice. During the later part of the series in which the atrial appendage was addressed, the appendage was excised rather than excluded in situ because of recent concerns about the long-term durability of exclusion compared with excision. However, this concern was not thought to be relevant to this study because the risk of postoperative AF-related CVA was followed to only 30 days. Future studies should incorporate transesophageal echocardiographic evaluation of the completeness of atrial exclusion or excision, as well as longer-term follow-up to ensure the durability of the exclusion and long-term decrease in postoperative AF-related CVA.

This study is limited by its retrospective and nonrandomized nature. Multivariable analysis was not feasible because of the low incidence of postoperative CVA, including postoperative AF-related CVA. However, there was a more than 10 -fold decrease in the rate of postoperative CVA in patients who developed postoperative AF (from $1.1 \%$ to $0.1 \%$ ) when the LAA was ligated. Although the LAA exclusion group was significantly different than the non-LAA ligation group according to the history of CHF and history of hypertension, the significance of this finding was nullified after propensity score matching.

In addition, the anticoagulation strategy in patients developing $\mathrm{AF}$ was not specifically addressed in the current study. Although no specific protocols were used, it was the author's (N.B.) practice, before implementing routine LAA exclusion, to start administering unfractionated heparin to all patients who developed AF that persisted for more than 24 hours after the initial onset. Once routine LAA exclusion was implemented, a less aggressive anticoagulation strategy for postoperative $\mathrm{AF}$ was used, with most patients not being anticoagulated at all unless they were discharged in AF. Despite this less aggressive strategy, there were still fewer postoperative AF-related CVAs in the patients who underwent LAA exclusion. Because none of the postoperative CVAs were hemorrhagic, the more aggressive use of anticoagulation before routine LAA ligation was not a factor in the increased CVA rate in the non-LAA ligation group.

\section{CONCLUSIONS}

Although the findings in this study are encouraging, the impact of routine LAA ligation in cardiac surgery needs to be further investigated in a more controlled fashion. The study results show that a prospective and randomized trial to further demonstrate the safety and efficacy of routine LAA exclusion is necessary and justified. Although routine LAA ligation has been shown to be a safe procedure in this series and commercial devices are now available specifically for LAA occlusion, which have been shown to be safe and effective for this purpose, such a trial should be performed before recommending LAA exclusion or excision as a routine adjunct to cardiac surgical procedures.

\section{References}

1. Almassi GH, Schowalter T, Nicolosi AC, Aggarwal A, Moritz TE, Henderson WG, et al. Atrial fibrillation after cardiac surgery. Ann Surg. 1997; 226:501-13.

2. Aranki SF, Shaw DP, Adams DH, Rizzo RJ, Couper GS, VanderVliet M, et al. Predictors of atrial fibrillation after coronary artery surgery: current trends and impact on hospital resources. Circulation. 1996;94:390-7.

3. Auer J, Weber T, Berent R, Ng CK, Lamm G, Eber B. Risk factors of postoperative atrial fibrillation after cardiac surgery. J Card Surg. 2005;20: 425-31.

4. Blackshear JL, Odell JA. Appendage obliteration to reduce stroke in cardiac surgical patients with atrial fibrillation. Ann Thorac Surg. 1996;61:755-9.

5. Chung MK, Asher CR, Dykstra D, et al. Does post-operative atrial fibrillation predispose to stroke, embolic events, or death after coronary artery bypass grafting? Circulation. 1995;92:I-644.

6. Lauer MS, Eagle KA, Buckley MJ, DeSanctis RW. Atrial fibrillation following coronary artery bypass surgery. Prog Cardiovasc Dis. 1989;31: 367-78.

7. Ommen SR, Odell JA, Stanton MS. Current concepts: atrial arrhythmias after cardiothoracic surgery. N Engl J Med. 1997;336:1429-34.

8. Crystal E, Lamy A, Connolly SJ, Klein P, Hohnloser SH, Semelhago L, et al. Left atrial appendage occlusion study: a randomized clinical trial of left atrial appendage occlusion during routine coronary artery bypass graft surgery for long-term stroke prevention. Am Heart J. 2003;145:174-8.

9. Johnson WD, Ganjoo AK, Stone CD, Srivyas RC, Howard M. The left atrial appendage: our most lethal human attachment! Surgical implications. Eur J Cardiothorac Surg. 2000;17:718-22.

10. Syed TM, Halperin JL. Left atrial appendage closure for stroke prevention in atrial fibrillation: state of the art and current challenges. Nat Clin Pract Cardiovasc Med. 2007;4:428-35.

11. Odell JA, Blackshear JL, Davies E, Byrne WJ, Kollmorgen CF, Edwards WD, et al. Thoracoscopic obliteration of the left atrial appendage: potential for stroke reduction? Ann Thorac Surg. 1996;61:565-9.

12. Park JW, Leithauser B, Gerk U, Vrsansky M, Jung F. Percutaneous left atrial appendage transcatheter occlusion for stroke prevention in atrial fibrillation: 2-year outcomes. J Invasive Cardiol. 2009;21:446-7.

13. Fuller JA, Adams GG, Buxton B. Atrial fibrillation after coronary artery bypass grafting: is it a disorder of the elderly? J Thorac Cardiovasc Surg. 1989;97: $821-5$.

14. Verhaert D, Puwanant S, Gillinov AM, Klein AL. Atrial fibrillation after open heart surgery: how safe is early conversion without anticoagulation? J Am Soc Echocardiogr. 2009;22:212.e1-3.

15. Taylor GJ, Malik SA, Colliver JA, Dove JT, Moses HW, Mikell FL, et al. Usefulness of atrial fibrillation as a predictor of stroke after isolated coronary artery bypass grafting. Am J Cardiol. 1987;60:905-7.

16. Bonow RO, Carabello BA, Chatterjee K, et al. ACC/AHA 2006 guidelines for the management of patients with valvular heart disease: a report of the American College of Cardiology/Guidelines (writing committee to revise the 1998 guidelines for the management of patients with valvular heart disease) developed in collaboration with the Society of Cardiovascular Anesthesiologists endorsed by the Society for Cardiovascular Angiography and Interventions and the Society of Thoracic Surgeons. J Am Coll Cardiol. 2006;48:e1-148. 
17. Katz ES, Tsiamtsiouris TT, Applebaum RM, Schwartzbard A, Tunick PA, Kronzon I. Surgical left atrial appendage ligation is frequently incomplete: a transesophageal echocardiographic study. J Am Coll Cardiol. 2000;36:468-71.

\section{Discussion}

Dr David A. Fullerton (Aurora, Colo). Although this is a retrospective study, the results are intriguing and suggest that there may be something we can do to improve the outcomes of our patients. This was a retrospective study of a heterogeneous group of patients. Do you think there may be subsets of patients for whom this would be most appropriate, for instance, those undergoing valve operations as opposed to just coronary bypass operation?

Dr Kim. Our intention was to provide ligation to everybody to decrease the risk of postoperative CVA in the patients who developed postoperative AF. On the basis of our results, in the patient who has a high risk of developing postoperative AF, the ligation should be recommended or considered to prevent possible postoperative CVA as a result of developing postoperative AF.

Dr Fullerton. One of the confounding variables that might influence the data was the anticoagulation management of patients who may have gone into AF postoperatively. Were there differences in the anticoagulation management between the 2 groups?

Dr Kim. To review the details of anticoagulation administration to the patients who developed postoperative AF was difficult in a chart review; therefore, these were excluded in our study. However, the practice protocol was if the patient developed postoperative AF and it persisted for 24 hours, anticoagulation was initiated at the beginning of the study. Again, because this was a retrospective chart review, a decreased incidence of postoperative AF-related CVA was observed. The 24-hour rule was not followed as strictly, unless the patient developed symptoms of AF.
Dr Richard Shemin (Los Angeles, Calif). I have 2 brief questions. The first is about the definition of stroke. Was this done including transient ischemic attacks? Was this within 30 days or just in hospital?

Dr Kim. It was within the postoperative hospital stay and any encounter until postoperative day 30. All the CVAs that were documented as positive postoperative CVA were confirmed with an imaging study. A few patients had postoperative CVAs that were not related to the postoperative AF, and these patients were found to have carotid artery thrombosis or occlusion or other unknown reasons that could cause the CVA. These cases were not studied in depth and not included in the study.

Dr Shemin. So these were completed strokes, not transient ischemic attacks.

Dr Kim. That is correct.

Dr Shemin. Were there any complications of the routine ligation? You did an excision and 2-layer closure. Any returns to the operating room for bleeding?

Dr Kim. There was zero take-back or second-look for bleeding from the ligation. Part of it was that the appendage was excised, and if it was not completely sealed we would have known before closing the chest. There was one incident in which the assistant was pulling the atria too hard, resulting in a small tear, but it was repaired immediately. There were no other complications.

Dr Shemin. Do you have any explanation why the test or ligation group had such a higher incidence of postoperative AF compared with the other group?

Dr Kim. The current trend is that the number of CABGs is slowly decreasing as cardiologists are performing more interventions, and at the same time the number of valve surgeries is increasing. According to our study, the valve surgery was actually statistically significant in the chi-square test. But this was not studied well and needs to be further investigated. 